\title{
Das reine Gold der «tendenzlosen» Analyse, die Kupferlegierung der "suggestiven" Therapie oder: mit welchen Rohstoffen operiert die Freudsche Analyse?
}

\author{
Rony Weissberg (Zürich)
}

Zusammenfassung: Der Autor wendet sich gegen eine kategorische Trennung und Unterscheidung von Psychoanalyse und Psychotherapie und meint, dass eine grundlegende Unschärfe, eine Ambivalenz zum Wesen der analytischen Erfahrung, letztlich aber auch zur Freudschen Theorie und Praxis gehört, dass diese sowohl analytische, entbindende, wie synthetische, bindende Aspekte umfasst. Gegenüber einer Dichothomisierung und einer Idealisierung der einen oder anderen Methode gehe es um das Begehren des Analytikers, um seine Ausrichtung auf das Unbewusste. Dabei steht weniger die Frage der Suggestion im Zentrum, als die eines spezifischen Glaubens, dem fundamentalen Unglauben, nämlich: dass wir «nicht Herr im eigenen Haus sind». Es sei dieser Unglaube, der uns ermöglicht, am Unbewussten festzuhalten, aus dem das Wesen des Subjekts hervorgeht. Allerdings müssen wir sehen, dass dieses Denken und Handeln in einer Tradition steht, die heute stark konkurriert und auf die Probe gestellt wird durch andere Werte und gesellschaftliche Ansprüche.

Schlüsselwörter: Abstinenz, Begehren des Analytikers, Glauben, Neutralität, Paradigma der Unschärfe, psychoanalytische Therapie, Suggestion, Unbewusstes

«Sie wissen, wir waren nie stolz auf die Vollständigkeit und Abgeschlossenheit unseres Könnens undWissens; wir sind, wie früher so auch jetzt, immer bereit, die Unvollkommenheit unserer Erkenntnis zuzugeben, neues dazuzulernen und an unserem Vorgehen abzuändern, was sich durch besseres ersetzen lässt.» Und weiter heisst es, dass es ihn reizt, «den Stand unserer Therapie zu revidieren ...» (Freud 1919a: 241).

Mit diesen Worten beginnt der Vortrag, den Freud 1918 in Budapest gehalten hat und der den Titel trägt «Wege der psychoanalytischen Therapie» und darin finden wir auch die häufig zitierte Metaphern vom reinen Gold der Analyse, das reichlich mit dem Kupfer der direkten Suggestion zu legieren sei. Die in diesem 
Aufsatz Freuds angesprochene Unterscheidung der therapeutischen Haltung in Neutralität einerseits und Suggestion andererseits bildet einen Ausgangspunkt, eine Ausgangsdifferenz dieses Zyklus hier am Psychoanalytischen Seminar Zürich. Dieser beschäftigt sich mit dem Unterschied von Psychoanalyse und Psychotherapie, oder wie auch schon formuliert, von Fundis oder Philosophen einerseits und Realos, Heilern, ja vielleicht gar Lieben und Netten andererseits. Wir haben es also mit einem Verhältnis zu tun, dass nicht ohne Entwertung ist, dabei herrscht keinesfalls Gewissheit darüber, was im einen Falle jeweils getan und im anderen Falle gelassen wird, ob da analysiert und dort synthetisiert wird, dass da mit Neutralität und dort mit Suggestion gearbeitet wird, dass man da auf Erkenntnis und dort auf Heilung ausgerichtet ist, wer letztlich nett ist und wer weniger. Wie ich zeigen werde, denke ich, dass gerade eine dezidierte Unterscheidung und Ausgrenzung dieser doppelgesichtigen Aspekte ein Symptom im analytischen Verständnis darstellt und dass es genau jener Unschärfe widerspricht, die meines Erachtens wesentlich ist für das psychoanalytische Denken und den psychoanalytischen Prozess.

Wie Sie wissen, steht dieser Zyklus im Zusammenhang mit einer schwierigen Situation, in der sich das Seminar befindet. Mehr und mehr steht es unter dem Druck, eine offiziell anerkannte Weiterbildung in Psychotherapie anzubieten, die sich sowohl bezüglich dessen, wie Weiterbildung zu geschehen hat, als auch bezüglich dem, was unter Therapie verstanden wird, an Standards orientiert, die zum Teil weit entfernt sind von der psychoanalytischen Tradition. Natürlich steht diese Entwicklung in einem gesellschafts- und gesundheitspolitischen Kontext und all dies bildet den komplexen Hintergrund dieses Zyklus, in dem ich mir das letzte Vortragsdatum geben liess, in der Absicht, mir genügend Zeit zu lassen, dies zu verfassen, was ich ihnen hier vortrage. Damit spreche ich nun aber auch nach all den Vorrednern, nach all dem schon Gesagten, mache nochmals den Versuch, mich der Frage, mich unserer Frage zu widmen, nicht einfach nach der Ästhetik einer Differenz von Psychoanalyse und Psychotherapie, sondern letztlich der Frage, wohin geht es mit der Psychoanalyse, wie geht es weiter?

In den vorangegangenen Vorträgen dieses Zyklus war wiederholt die Rede von der Gegenüberstellung von Analysieren, als dem Herausschälen einzelner unbewusster Motive, und von Synthetisieren, als dem Zusammenfügen solcher Aspekte in die Gesamtpersönlichkeit, von der Gegenüberstellung von Erkenntnis einerseits und Suggestion und Heilung andererseits. Um bereits Gesagtes nicht allzu sehr zu wiederholen, werde ich das im Titel erwähnte psychoanalytische Gold und psychotherapeutische Kupfer etwas weniger prominent behandeln, als 
ich es vorgesehen hatte. Ursprünglich wollte ich diese Dualität von tendenzloser Neutralität und manipulativer Suggestion eingehend beleuchten; ich wollte insbesondere aufzeigen, wie wenig sich Freud selbst an seine eignen Vorgaben und Idealbilder hielt, wie stark sein psychoanalytisches Handeln Tendenzen hatte, z. B. geprägt war von seiner Neugier, seinem Forscherglauben, seiner Subjektivität, ja wie sehr die jeweilige Dauer, das Setting aber auch Freuds Interventionen von Analyse zu Analyse variieren (vgl. z. B. May 2007).

Trotz dieser evidenten Freudschen Praxis gab es bekanntlich innerhalb der Psychoanalyse eine starke Ausrichtung auf Ideale, wie z. B. das der Neutralität, der Standardtechnik, Ideale, die später z.T. äusserst dogmatisch verfochten wurden. Gegenüber einer solchen Ausrichtung an stark formalisierten Idealen von dem, was Psychoanalyse zu sein hat, geht es mir im Folgenden um das, was ich die hochspezifische Dynamik von Subjektivität und Leidenschaft im psychoanalytischen Prozess bezeichnen möchte. Es wird mir also weniger um die Geschichte der beiden ungleichen Geschwister Psychoanalyse und Psychotherapie gehen, sondern um das, was ich als Freudsche Grundhaltung erachte. Und insofern geht es mir zum einen darum, das Prekäre, das vielleicht Tabuisierte dieser Erfahrungswissenschaft zu verdeutlichen. Zum Anderen geht es mir um eine Annäherung an eine Dialektik von Trieberfahrung und metapsychologischer Reflexion, einer Erfahrung am Rande von Sprache und Angst, und zugleich einem Denken, das diese Erfahrung in höchster Abstraktion zu erfassen sucht. Aus diesem Verständnis des psychoanalytischen Geschehens heraus geht es mir letztlich um das Begehren des Analytikers, das für diesen Prozess steht, das diesen Prozess letztlich in starkem Masse trägt. Diesen Fokus möchte ich im Folgenden umkreisen, um dann letztlich auf das zu kommen, was ich im Titel andeute: auf die Kräfte, die Rohstoffe, die den psychoanalytischen Prozess vorantreiben. Ich meine damit den Trieb als der spezifischen Realität des Unbewussten in der Psychoanalyse, ein Konzept, das unter dem Einfluss eines gesellschaftlich geprägten Wirklichkeitsverständnis immer mehr zu verschwinden droht. Begehren und Trieb stehen aber noch in Bezug zu einem weiteren Thema, das ich nicht vermeiden möchte und das in unserer Zeit der Auf- und Abgeklärtheit, unserer Zeit des autonomen Subjekts vielleicht noch heikler ist als das der Suggestion; ich meine hier die Sache des Glaubens. Doch dazu später.

Damit komme ich auf das Zitat Freuds zurück, mit dem ich begonnen hatte: «Sie wissen, wir waren nie stolz auf die Vollständigkeit ...», welches damit schliesst, dass es ihn reizt, «den Stand unserer Therapie zu revidieren ...». Lassen wir einmal offen, wie bereit Freud tatsächlich war, seine Therapie zu revidieren, sicherlich aber ist es meines Erachtens eine seiner grössten Qualitäten, dass er eine 
Fähigkeit besass, sein Unwissen, seine Neugier, aber auch die Unvollkommenheit, die Unabgeschlossenheit in seinem Denken und Konzipieren aufrechtzuerhalten. Er für sich schien dies zu können, er konnte sich hinterfragen, Schwachstellen diskutieren, sich kritisieren. Es geht hier nicht zuletzt um eine bedeutsame, ja radikale Frage des Narzissmus, um die Möglichkeit Inkonsistenzen offen zu lassen, Grenzen und fehlendes Wissen anzuerkennen und stehen lassen zu können. Wir wissen es ja wohl alle, dass es nicht so simpel ist, sich dem Unbewussten in diesem Sinne auszusetzen. Die Konfrontation mit Unwissenheit, Unfassbarkeit, Verunsicherung aufgrund der «unbewussten Realität» gehört zum Wesen der Psychoanalyse. Die Geschichte allerdings dieser Konfrontation, d.h. der «State of mind» der Psychoanalyse, ihre Radikalisierungen respektive Anpassungen, wird von der äusseren Realität mitgeprägt, davon, wie sehr Prinzipien wie Machbarkeit, Funktionalität, Gewissheit, Messbarkeit vorherrschen, davon, dass Gratifikationen winken oder Verordnungen gelten, wie z. B. wenn man in der Lage sein müsste, spätestens nach sechs Sitzungen über Diagnose und Prognose einer Therapie zu befinden. Hier stellt sich dann die Frage, wie sehr man noch in der Lage ist, zur Sache des Unbewussten zu stehen.

Ich kehre zurück zu Freuds Aufsatz «Wege der Psychoanalytischen Therapie» (Freud 1919a). Dabei zeigen sich erstaunlichen Passagen, ja man könnte an gewissen Stellen gar meinen, man hätte hier ein Instruktionspapier für werdende Verhaltenstherapeuten in den Händen. Zunächst aber wendet sich Freud gegen den Anspruch, Analytiker müssten nicht nur analysieren, sondern auch Synthetisieren und meint dazu, dass der Zwang zur Vereinheitlichung, also zur Synthese, im Psychischen ohnehin bestünde und nicht der speziellen Hilfe des Analytikers bedarf. Die Entwicklung «unserer Therapie» gehe gemäss Freud vielmehr in eine ganz andere Richtung, nämlich in der Art, die Ferenczi mit Aktivitäten des Analytikers (a.a.O.: 243) bezeichnet habe und diese Aktivitäten gelten wieder der Bewusstmachung des Verdrängten und dem Aufdecken der Widerstände; nichts neues also unter der Sonne und man könnte sich je nach Gesinnung beruhigt zurücklehnen oder das Buch enttäuscht beiseite legen, denn Freud wiederholt hier das, was er mehrfach zuvor schon als klassischen Zugang zum Unbewussten dargelegt hatte. Dann aber kommen zwei, drei Gedanken zur therapeutischen Arbeit, die keineswegs der standardisierten Freud-Rezeption entsprechen. Dies betrifft zunächst einmal sein radikaler Umgang mit der Abstinenz, mit der Versagung: gemeint ist die Vermeidung von Ersatzbefriedigungen und im Besonderen der Befriedigung von unerfüllten Wünschen, wie sie sich in die Übertragungsbeziehung einschleichen können. Für Freud geht es dabei weder 
um Deutungen noch um den schweigenden Analytiker; vielmehr spricht er davon, dem Kranken gerade die wichtigsten Befriedigungen zu versagen (a.a.O.: 245 f.), damit es wirklich zu einer Besserung kommen kann. Eine ähnliche Radikalität finden wir in gewissen Interventionen Lacans, die auf die Konfrontation mit der zentralen, phantasmatischen Angst ausgerichtet sind, Interventionen die immer wieder sehr ablehnend von der analytischen Gemeinde aufgenommen wurden. Ich denke dabei insbesondere an die Funktion des Schnitts, auf die ich weiter unten noch zu sprechen komme.

Einen weiteren überraschenden Punkt in Freuds Artikel ist sodann das, was ich seinen störungsspezifischen Zugang nennen möchte. Nachdem er nochmals erwähnt, dass es in der Psychoanalyse nicht darum gehen kann, den Anderen, dessen Schicksal, nach dem eigenen Ideal zu formen, erwähnt er, dass es nicht zu vermeiden sei, «auch Patienten anzunehmen, die so haltlos und existenzunfähig sind, dass man bei ihnen die analytische Beeinflussung mit der erzieherischen vereinigen muss» (a.a.O.: 246 f.), ein Zugang, der z. B. im Umgang mit Psychotikern sicherlich nicht von der Hand zu weisen ist. Freud fährt dann fort, dass wir genötigt seien, die «verschiedenen Krankheitsformen (...) nicht durch die nämliche Technik» zu erledigen. So könne man beispielsweise eine Phobie nicht wie eine Hysterie behandeln. Wenn der Phobiker z. B. vermeide, in die Aussenwelt zu gehen, müsse man ihn dazu bringen, wieder auf die Strasse zu gehen und sich mit der Angst zu konfrontieren. Ähnliches sagt er zu Fällen von Zwangshandlungen, wo er meint, dass sobald die Kur selbst zum Zwang geworden sei, dieser künstliche Zwang als Gegenzwang gegen den Krankheitszwang einzusetzen sei. Dies seien, so Freud, allerdings nur einige Proben der neuen Entwicklung unserer Therapie (a.a.O.: 248).

Und erst jetzt, nach all diesen doch recht konkreten Darlegungen zu den Möglichkeiten einer aktiven Psychoanalyse, erst jetzt folgt der Hinweis auf das Massenelend in Folge des ersten Weltkriegs - der Vortrag wurde, wie erwähnt, 1918 gehalten -, und im weiteren das berühmte Zitat, dass davon handelt, «(...) dass wir genötigt sein werden, in der Massenanwendung unserer Therapie das reine Gold der Analyse reichlich mit dem Kupfer der direkten Suggestion zu legieren(...).» Und er meint auch, dass hier auch die Hypnose wieder ihre Anwendung finden könnte, um schliesslich den Artikel mit der apodiktischen Sentenz zu beenden: «(...) aus welchen Elementen sie (die Massenanwendung [R.W.]) sich zusammensetzen mag, ihre wirksamsten und wichtigsten Bestandteile werden gewiss die bleiben, die von der strengen, der tendenzlosen Psychoanalyse entlehnt worden sind» (a.a.O.: 249). Es ist erstaunlich, wie sehr diese «Gold-Kupfer-Passage» immer 
wieder aus dem Kontext gerissen wurde und zu allerlei Interpretationen herhalten musste. Dass seine zumindest ebenso markanteren Passagen über einem möglichen Wandel «unserer Therapie» in den vergangenen 90 Jahren kaum Erwähnung fanden und letztlich auf wenig fruchtbaren Boden stiessen, bleibt bemerkenswert. Vielleicht hat es mit Freuds Ambivalenz zur «legierten» Therapie zu tun, dem Ideal der Neutralität: Freud jedenfalls mag sie nicht, mag sie nicht mehr die Suggestion. Und er sagt dies, obschon er weiss, dass sich etwas Suggestives nicht ganz aus dem Analytischen vertreiben lässt, denn er schreibt 1916, am Ende seines ersten Vorlesungszyklus: «(...) dass wir unsere Technik der Hypnose nur aufgegeben haben, um die Suggestion in der Gestalt der Übertragung wiederzuentdecken» (Freud 1916/17: 429).

Und ohnehin hat Freud, wie Anton Fischer (2007) in seinem Vortrag aufzeigt, letztlich wenig übrig fürs Massenelend, für das ja die Suggestion gedacht war. Die Psychoanalyse war für Freud eine elitäre Angelegenheit von Reichen, die es sich leisten konnten. Diesbezüglich geht es um einen privatwirtschaftlichen Vorgang, unabhängig von der sozioökonomischen Realität. Es fehlte Freud ja auch nicht an Verachtung für das, was er «wertlose Individuen» nannte. Freud hatte sein Klientel, das sich sozusagen unter seine Fittiche begab, um sich den Orden des Ödipus verleihen zu lassen, wie Fischer schreibt. In diesem Sinne gab es für ihn auch keine materielle Notwendigkeit, die Technik zu revidieren. Allein: das Verleihen von Orden ist ja wohl nicht ganz ohne Suggestion zu denken?! Er mag sie nicht, die Suggestion, und er gebraucht sie und zwar nicht in geringem Masse, und genau darin sehe ich Freuds Ambivalenz, in seiner Pragmatik, seiner öffnenden Unschärfe: Und dies meint keine fehlende Prägnanz, sondern eine ständige Suche.

In diesem Sinne muss man somit Freuds Verhältnis zur Psychotherapie als ambivalent bezeichnen. So wenig er sich am Ende für das Massenelend einsetzte, so sehr er letztlich Ferenczis «Schmusetherapie» verurteilte und auf strengen Behandlungsregeln bestand, so sehr war er andererseits in seinem eigenen Vorgehen innovativ. Es macht den Eindruck, dass er kein Setting und keine Technik verabscheute, wenn es darum ging, die Sache des Unbewussten zu erforschen. Wenn ich den Aufsatz von 1918 auf einen Punkt bringen möchte, so würde ich sagen, dass diese Freudsche Ambivalenz der Psychotherapie gegenüber darin zum Ausdruck kommt, dass er mit Nachdruck auf der Abstinenz und Neutralität besteht, wie er mit Nachdruck auf die Sache der Aktivität des Analytikers verweist. Dass letzteres in der Rezeption Freuds so randständig wahr- und aufgenommen wurde, hat wohl damit zu tun, dass man ihn gerne auf eine eindeutige Position 
verpflichten will, dass man die von mir postulierte Ambivalenz, Unschärfe ausmerzen möchte, nicht haben will. Grundsätzlich aber vermute ich, dass Freud im Zusammenhang mit den von ihm verfassten technischen Regeln mit der eigenen Vernunft und Kreativität der Analytiker rechnete, dass diese, ausgehend von einer festen Struktur, einer relativ rigiden klinischen Theorie, selbst innovativ auf die je spezifischen Situationen reagieren würden. Und dürfen wir nicht annehmen, dass dies auch geschieht? Zudem vermute ich, dass Freud vermeiden wollte, dass seine Methode als Legierung zu einer Psychotechnik verkomme, wenn Psychotherapie zu sehr eigenständig theoretisiert und unterrichtet würde, eine Gefahr, die heute sicherlich besteht.

Gegenüber dieser Ambivalenz von Freud, die etwas offen lässt und die etwas von der Unschärfe aufrechterhält, führt die Verunsicherung, die die Beschäftigung mit dem Unbewussten mit sich bringt, immer wieder zu ideologischen und zum Teil dogmatischen Konzeptionen der Psychoanalyse. Ich möchte in unserem Zusammenhang auf zwei solche Phänomene zu sprechen kommen: zum einen auf die bereits erwähnte Standardtechnik und der damit relativ eng verbundenen Ich-Psychologie, die sich bekanntlich in den USA der 50er Jahre etablierte und zum anderen auf das, was man als subversives Denken bezeichnen kann, das an unserem Seminar in den späten 70ern und frühen 80ern eine bedeutende Rolle spielte.

In diesen Jahren erwies sich die Psychoanalyse häufig als wesentlicher Bestandteil der Ideologie der 68er und galt nur schon deshalb als subversiv. Dieses politischeVerständnis von Psychoanalyse wurde lange auch am PSZ gepflegt. Dabei reichte linkes, politisches Handeln, um als subversiv zu gelten, während gleichzeitig eine eher reaktionäre ich-psychologische Psychoanalyse portiert wurde. Nachträglich muss man hier von einem Missverstehen bezüglich der Freudschen Sache ausgehen: Sicherlich war Freud immer ein Denker, der sich mit kulturellen und gesellschaftlich Fragen beschäftigte, aber wie z. B. auch aus seinem Verhältnis zum Massenelende deutlich wird, war er mit Sicherheit kein Linker und auch kein Subversiver im politischen Sinne. Allerdings halte ich sein Denken dennoch für grundlegend subversiv, und zwar wegen seiner Fähigkeit, gegen alle Widersacher, gegen alle Verunglimpfungen am «anderen Schauplatz» festhalten zu können; aufgrund seines Insistierens aufder Sache des Unbewussten, der Verdrängung und der infantilen Sexualität. Und vor dieser Herausforderung stehen wir auch heute noch, wo es darum geht, im heutigen gesundheits- und gesellschaftspolitischen Umfeld, das wenig vom Unbewussten wissen will, die Sache der Psychoanalyse weiterzudenken. 
Damit möchte ich auf die andere zuvor angesprochene dogmatische Konzeption zu sprechen kommen, auf die Standardtechnik. Diese «ideale Technik» bestand in einem Anspruch auf Neutralität des Analytikers, welcher das Attribut der Wissenschaftlichkeit zugemessen wurde. Diese Technik setzte allein auf die Deutung und auf den ansonsten schweigenden Analytiker, der in erster Linie keinen Fehler machen sollte. Diese Tendenz zur Fetischisierung der Deutung, ähnlich wie der Hang zur Fetischisierung der Technik oder des Settings könnte, analog dem klinischen Fetisch, verstanden werden als Versuch der Vervollkommnung, der Verleugnung der Kastration, des Erschaffens eines absoluten Wissens, der Absicherung. Martin Kuster (in diesem Heft) schreibt im Zusammenhang mit der Standardtechnik: «Ich behaupte, dass ein Problem dieser Idealtechnik war, dass sie nicht auf etwas Früherem, Vorausgegangenem aufbauen konnte - wie Freud auf der Hypnose» - und er meint weiter, «dass Strenge und Perfektheit eine daraus resultierende, folgenreiche rein amerikanische Erfindung sei» (29). Soweit Kuster. Man könnte sagen, diese Idealtechnik sei die Ausgestaltung der Psychoanalyse im amerikanischen Puritanismus. Damit möchte ich zweierlei andeuten: zum einen, dass Erscheinungen in der Psychoanalyse immer in einem spezifischen Kontext zu denken sind, was zwar selbstredend ist, aber immer wieder in Vergessenheit gerät, wenn von den eigenen Haltungen hier und heute die Rede ist. Zum anderen, dass die Psychoanalyse existentiell auf einem wichtigen Prinzip beruht, nämlich dem Anlehnungsprinzip: das sie sich in Anlehnung an etwas entwickelt, so wie die Sexualität sich anlehnt an die Selbsterhaltung oder das Kind an die Eltern.

In unserem Kontext, im Zusammenhang mit der Frage zur Zukunft der Psychoanalyse, möchte ich im Folgenden weniger von Anlehnung als von Tradition sprechen, der Tradition innerhalb derer sich jemand an das anlehnen kann, was tradiert wird. Ich denke, dass es für die weitere Geschichte des Seminars, aber auch für die Entwicklung der Psychoanalyse und ihrer Therapie wesentlich ist, wie wir diese Tradition weiter leben und entwickeln. Ich möchte diese Tradition an einigen wenigen Punkten nuancieren. Dabei geht es mit vor allem um das Verhältnis zum Wissen einerseits und um das Verhältnis zur Kraft, zum Trieb andererseits, sowie um die Verknüpfung dieser beiden Aspekte.

1. Zunächst zur Form der Tradierung: Bislang war die Psychoanalyse gewissermassen ein Kunsthandwerk im Sinne einer Tradition, in der man sein Können mit und an Vorbildern erwarb. So schreibt Freud zur Ausbildungsanalyse: «Ihre Leistung ist erfüllt, wenn sie dem Lehrling eine sichere Überzeugung von der Existenz des Unbewussten bringt(...)» (Freud 1937: 388). So aber wie das traditionelle Handwerk am verschwin- 
den ist, scheint auch die Bereitschaft zu verschwinden, sich eingehend mit der komplexen Theorie und der intensiven Praxis der psychoanalytischen Erfahrung auseinanderzusetzen. Damit allerdings schwände auch das Erfahrungspotential und damit auch das Erkenntnispotential der Psychoanalyse.

2. Die Psychoanalyse ist eine Erfahrungswissenschaft die auf induktiver Erkenntnisgewinnung basiert, d.h. Erkenntnisse werden ganz im Sinne des Freudschen Diktums von «Forschen und Heilen» aus dem «Einzelfall» abgeleitet und entwickelt. So zumindest die Ideologie der Psychoanalyse. Ich denke, dass die Psychoanalyse heute zu einem schönen Teil eine Verwaltungswissenschaft geworden ist, wobei häufig alte Erkenntnisse repliziert und zitiert oder einer positivistischen Empirie und phänomenologischen Diagnostik zur Verfügung gestellt werden. Was dabei verloren geht ist das Erforschen aktueller, veränderter unbewusster Strukturen.

3. Der psychoanalytische Prozess ist ein hochspezifischer subjektiver Akt, ein Risiko, ein sich einer Erfahrung Aussetzen. Es geht dabei um eine grundlegend nicht-reziproke Konstellation, in der wir einen spezifischen Platz einnehmen, entweder als derjenige, an den die Rede gerichtet ist und der dem «Anderen» Sprechen seine Aufmerksamkeit widmet, oder als der, der selbst Subjekt des Sprechens ist.

4. Das analytische Denken zeichnet sich durch ein Paradigma der Unschärfe oder des doppelten Diskurses aus: Es kennt ein stetes Kippen: wo es sich der Erklärung nähert, wird es zu Verstehen und umgekehrt. Paul Ricoeur (1969) spricht in diesem Zusammenhang von einer gemischten Rede, die die Basis der Psychoanalyse sei und nennt die eine naturwissenschaftliche, erklärende Dimension der Psychoanalyse Energetik, die andere, die geisteswissenschaftliche, verstehende Dimension nennt er Hermeneutik. Dabei macht Ricoeur auch den vexierbildhaften Wechsel deutlich, wenn er schreibt: «Vielleicht liegt gerade im Setzen des Wunsches die Möglichkeit, von der Kraft zur Sprache überzugehen, aber auch die Unmöglichkeit, die Kraft völlig in die Sprache einzubringen» (Ricoeur 1969: 81). Ein ähnliches Wechselverhältnis besteht meines Erachtens zwischen Erkennen und Heilen, auch hier gibt es eine Kippbewegung, insofern Heilung in Erkenntnis und Erkenntnis in Heilung übergehen kann - oder eben auch nicht.

5. Die zuvor erwähnte Unmöglichkeit zeigt sich z.B. auch im Kontext von Trauma und Scham, darin, wie Trieb, Überflutung und Symbolisierung in einem Unverhältnis zueinander stehen können, das den psychoanaly- 
tischen Prozess immer wieder an die Grenze treibt, an die Grenze von Erfahrungsmöglichkeit und Sprachfindung. Ganz in diesem Sinne hatte Freud bekanntlich den Trieb als Grenzbegriff zwischen Seelischem und Somatischem eingeführt. Dabei definiert er den Trieb zum einen als psychischen Repräsentanten «der aus dem Körperinnern stammenden, in die Seele gelangenden Reize». Zum anderen: ... als ein Mass der Arbeitsanforderung, die dem Seelischen infolge seines Zusammenhangs mit dem Körperlichen auferlegt ist» (1915c: 85), man könnte sagen, er definiert ihn als Kraft und Sinn.

Dieses Grenzgängerische, so faszinierend es klingen mag, ist zugleich auch die grosse Schwierigkeit der Psychoanalyse. Sowohl mit dem verpönten, verbotenen Gedanken als auch mit der traumatisierenden, schamhaften Überflutung sind wir ausserhalb von Konvention und Sprache, im Ungewissen, im Unsicheren, quasi «hors discours», einer «fremden» inneren und äusseren Realität ausgesetzt. Und an dieser Grenzhaftigkeit liegt es wohl, dass wir es in diesem Feld von Trieb und Struktur auch mit einer Metapsychologie zu tun haben, deren Komplexität immer wieder so schwer zu erfassen ist. Wir sind dem Unbewussten ausgesetzt, und die Theorie bietet auch nicht einfach einen sicheren Hafen. Dies ist der Kontext, in dem Begriffe wie Subversion und Begehren des Analytikers Sinn machen, wo aber auch die erwähnten rigiden Ideale und Dogmen ihre defensive Relevanz erhalten, nämlich dort, wo es darum geht, sich der äusseren politischen und diskursiven Macht aber auch den inneren Ängsten zu stellen. Und in diesem ganzen Spannungsfeld spielt dann das eine Rolle, was ich mit Rohstoff, dem Trieb und mit dem Glauben angedeutet habe.

In seiner 27. Vorlesung zur Übertragung schreibt Freud (1916/17), dass das Wissen vom Unbewussten, das wir haben und dem Patienten mitteilen, wenig nütze, denn es komme nicht an Stelle von dessen Unbewusstem, sondern daneben. Wir müssten das Unbewusste am Ort seiner Entstehung, am Ort der Verdrängung aufsuchen: die Verdrängung gelte es zu beseitigen. Doch wie soll dies geschehen. Ich zitiere Freud: «Mit welchen Triebkräften arbeiten wir denn in einem solchen Fall?» (Freud 1916/17: 420). Dazu meint Freud weiter unten, dass es nicht die intellektuelle Einsicht sei, die den Ausschlag in diesem Kampf gäbe, sondern die Übertragung, das Verhältnis zum Arzt. «Soweit seine Übertragung von positivem Vorzeichen ist, bekleidet sie den Arzt mit Autorität, setzt sie sich in Glauben an seine Mitteilung und Auffassung um» (1916/17: 428). Er fährt fort, in dem er den Glauben aus der Entwicklungsgeschichte des Subjekts ableitet und als Abkömmling der Liebe bestimmt. Er spricht vom Subjekt, das zunächst keiner Argumente bedurfte 
und meint dann, dass bei vielen Menschen Argumente ohne die Stütze von Liebe und Glaube ein Leben lang nichts gelten. Der Mensch sei intellektuell nur insofern zugänglich, wenn er ein Objekt libidinös besetzen kann und man müsse im Narzissmus eine Schranke für die Beeinflussbarkeit auch für die beste analytische Technik sehen. Die Suggestibilität sei somit nichts anderes als die Neigung zur Übertragung, und diese sei «von der Sexualität, von der Betätigung der Libido» (Freud 1916/17: 429) abhängig. Diese Textstelle zeigt nicht zuletzt die radikale Dekonstruktion eines zu simplen Verständnisses von Aufklärung. Sie verweist auf die Schwäche von Wissen und Wahrheit im Bezug auf gegenläufige, unbewusste Kräfte und die Notwendigkeit der Stütze des Intellekts durch die Liebe und den Glauben an die Person, der man, wie Lacan es formuliert, Wissen unterstellt.

Das bringt uns nochmals zurück zur Sache der Suggestion, die ja für Freud das zentrale Unterscheidungsmerkmal von Psychoanalyse und Psychotherapie ist. So sehr Freud eine Unterscheidung macht zwischen der hypnotischen Suggestion, die für ihn eine Kosmetik ist, insofern sie Symptome verbietet und Verdrängung stärkt, und die er von der analytischen Suggestion, d.h. von der Beeinflussung aufgrund von Übertragung und Glaube an den Arzt unterscheidet, so misstrauisch bleibt er auch gegenüber dieser Machtwirkung des Arztes und fordert, dass «zum Schluss einer analytischen Kur (...) die Übertragung selbst abgetragen sein (...)» (1916/17: 435) muss und dass erst dadurch die objektiven Ergebnisse der Kur erkennbar würden. Es gehört zum Schicksal der deutschsprachigen Psychoanalyse, dass sie sich mit dem Begriff der Suggestion sehr schwer tut. Aber auch für Lacan ist die Suggestion das, was die Unterscheidung von Therapie und Analyse ausmacht. Ausgehend von Freuds «Massenpsychologie und Ich-Analyse» sieht er das Problem der Suggestion in einem «Nicht-Getrennt-Sein» vom unerreichbaren Objekt a einerseits und vom Ich-Ideal andererseits und zwar personifiziert in der, auf diese Weise mit Macht ausgestatteten Person des Hypnotiseurs. Daher besteht er darauf, dass es in der Analyse nicht um die Identifikation mit dem Analytiker als Idealem, als Wissendem geht. Denn, was aufgrund der Unterstellung von Wissen, aufgrund des Glaubens in die Person des Analytikers die Übertragungsliebe auslöst, die, wie erwähnt, zur Stütze wird, mutiert andererseits zum Widerstand, zum Moment des Verschliessens des Unbewussten (Lacan 1964: 281 ff.). Das, was an dieser Stelle von der Seite des Analytikers den Prozess vorantreibt, ist dann nicht seine Anerkennung als Wissender, auch nicht sein Verstehen, sondern ganz simpel sein Zuhören, sein Vernehmen dessen, was sich aus dem Unbewussten des Analysanden artikuliert. Genau das ist gemeint beim hehren Lacanschen Diktum: das Begehren des Analytikers. 
Bevor ich die obigen Gedanken zur Suggestion mit der Sache des Glauben verknüpfe, möchte ich noch auf die von Peter Passetts in seinem Vortrag dargelegte Unterscheidung zwischen Analyse und Therapie zu sprechen kommen, bei der er sich auf den Todestrieb bezieht: Er betont zunächst, dass auch in Analysen die Hauptarbeit im Zusammensetzen von «disparaten Bruchstücken der Lebensgeschichte» besteht, sprich also im Synthetisieren, und schreibt dann: «(...) das Spezifische des analytischen Prozesses ist der Prozess der Loslösung aus alten Bindungen, des Entbindens und folgt damit der Tendenz dessen, was Freud den Todestrieb genannt hat» (Passett in diesem Heft). Somit gäbe es nur eine Analyse, während die psychoanalytische Psychotherapie, ausgerichtet auf die bindende Tendenz der Lebenstriebe, eine unter tausend verschiedenen Therapien sei. Spricht Freud im Zusammenhang mit der Analyse von der Notwendigkeit der Auflösung der Übertragung, geht es Lacan quasi um die Nicht-Identifikation, macht Passett das, worum es in der Analyse geht, am Entbinden fest. Aber alle diese Versuche, das Zentrale der Analyse zu erfassen, beziehen sich auf das Ende, die letzte Ausrichtung der jeweiligen «Behandlung» und bestimmen sozusagen im Nachhinein, was es "gewesen sein wird»: eine Analyse oder eine Therapie. Ich teile die Sichtweise, dass es nicht vorausgehende formale Kriterien sind, die darüber entscheiden, was wir tun, sondern innere, strukturelle Merkmale des Prozesses. Vielleicht haben wir mit der Unterscheidung von Gebunden und Entbunden, von Liebe und Sinnlichkeit, von Synthetisierung und Aufbrechen eine markante Beschreibung, um Psychoanalyse und Psychotherapie an ihren radikalen Polen zu unterscheiden, und diese Differenz ist sehr wohl wichtig für unsere Orientierung im Prozess. Letztlich finden wir aber auch hier wieder ein Wechselverhältnis, eine Unschärfe-Relation, wie ich sie zuvor beschrieben habe: Dass kein analytischer Prozess, kein Heilungsprozess ohne die Sache der Liebe auskommt, ist so selbstredend wie die Tatsache, dass ohne Momente, wie die der Entbindung, nichts aufbrechen kann, nichts Neues entstehen kann.

Aber nicht der Liebe, sondern dem Glauben wollte ich ein besonderes Augenmerk schenken. Wie erwähnt denke ich, dass der Begriff des Glaubens der Sache näher kommt als der der Suggestion. Zwar geht es im psychoanalytischen Prozess um Suggestibilität, um Beeinflussbarkeit, um Kräfte, die die Veränderung vorantreiben, aber nicht um willentliche Manipulation, um Suggestion. Der Glaube wurde bereits an zwei Stellen erwähnt: zum einen auf der Seite des Analytikers, im Zusammenhang mit der Tradierung der Psychoanalyse in der Ausbildungsanalyse. Man könnte sagen: der Glaube an die Erfahrung des Unbewussten. Des weiteren war vom Glauben als kindlicher Glaube die Rede: der Glaube an die ärztliche 
Hilfe als Stütze seiner Mitteilungen. Ich denke zudem, dass das, was ich zuvor als Begehren des Analytikers beschrieben, oder weiter oben also Subversion bezeichnet habe, nicht ohne den Glauben an die Sache des Unbewussten auskommt. Es ist die ethische Position, die davon ausgeht, dass das "Ich nicht Herr im eigenen Hause» ist, die Position, die nicht von einem selbstbewussten autonomen Subjekts ausgeht, und die darum ringt, «das unbewusste Subjekt zur Sprache zu bringen». Diese Position ist an einen Glauben geknüpft, insofern das Unbewusste zwar heftig erfahren werden kann, nicht aber als objektivierbares Wissen unmittelbar zu erschliessen ist. Dass dieser Glaube eine Nähe zum Religiösen hat, sich aber dennoch grundlegend davon unterscheidet, kann ich hier nur andeuten. Mit ihrem Glauben an das Begehren teilt die Psychoanalyse mit der Religion, dass sie kein eigentliches, messbares wissenschaftliches Objekt der Beobachtung hat. Aber insofern sie den Glauben selbst zum Objekt der Reflexion macht, unterscheidet sie sich wiederum radikal von der Religion. Boelderl (2002) nennt in Anlehnung an Lacan die spezifische psychoanalytische Form des Glauben «Anders-als-Glauben», und er meint damit einen fundamentalen Unglauben, einen Mangel an Glauben, das Fehlen einer letzten Gewissheit. Und gerade dies charakterisiert das, was man die nicht-kindliche Seite psychoanalytischen Glaubens nennen könnte, eine radikale Wirklichkeit des Glaubens, aus der das Wesen des Subjekts hervorgeht. Genau von dieser Art von «Anders-als Glauben» ist gemäss Boelderl das Verhältnis von Lacan zu Freud. Er schreibt: «Dem Lehrer in seiner Spur folgen heisst, ihm abtrünnig werden, ihn durch allerhöchste Treue zu verraten, (dass man) etwa indem man ihn wörtlich nimmt, versteht» (Boelderl 2002: 20). Glaube, so gedacht wäre dann das, was die Subjektivität in seiner radikalsten Form stützt, sie hervortreten lässt. Und diese Form des Glaubens/Unglaubens ist wiederum in engem Bezug zur Unschärfe, wie sie zuvor besprochen wurde.

Ich komme auf einen Fall zurück, über den ich an dieser Stelle schon einmal berichtet habe. Es handelt sich dabei um einen Bericht von Margaret Little, auf den Lacan in seinem Seminar $10 \mathrm{zu}$ sprechen kommt. Es geht um eine Frau, eine Kleptomanin, deren Vater von der Analysandin als selbstsüchtig und megaloman beschrieben wird. Die Mutter demgegenüber sei besitzergreifend, ausbeuterisch und streitsüchtig. Sie sei hart gezüchtigt worden von den Eltern, die sich eigentlich einen Sohn gewünscht hatten. Little berichtet, dass sie über Jahre mit ihren Deutungen nichts bewirken konnte: nichts habe sich am destruktiven Verhalten der Patientin geändert. Ausser den Diebstählen, die zunächst im Zusammenhang mit Treffen mit der Mutter, später im Kontext der Übertragung auftauchten, zeigte die Analysandin gefährliche Impulshandlungen; z. B. rannte sie manchmal aus der 
Praxis, ohne auf die Strasse zu schauen, und einmal wurde sie dabei angefahren. Die Beziehung der Analysandin zu ihr war von heftiger Ambivalenz und Kritik geprägt, sie habe sie despektierlich behandelt und ständig kritisiert, so z. B. wegen ihrer Praxiseinrichtung. Einmal sagte die Patientin zur Analytikerin, sie sei wie ihre Mutter, eine ganz hässliche Person. Gleichzeitig bemerkte die Analytikerin eine immer stärker werdende magische Kontrolle über sie: Die Patientin wollte sie immer bei sich haben, ertrug es aber kaum, als sie ihr einmal, sozusagen als «realem Menschen» im Theater begegnete.

Etwas begann sich zu ändern, als die Analytikerin es wagte, ihre Gefühle zum Ausdruck zu bringen und ihr sagte, es falle ihr schwer, bei den Geschichten über die Streitsucht ihrer Mutter wach zu bleiben; und noch deutlicher wurde sie, als sie zur Kritik an ihren Praxisräumen sagte: «I really don’t care, what you think about it.» Zu einer weiteren markanten Veränderung kam es dann, als sie einmal in Trauer, schwarz gekleidet in die Praxis kam. Die Analytikerin hatte bislang bei ihr noch nie eine Trauerreaktion wahrgenommen, selbst beim Tod des Vaters war keine Trauer erkennbar. Nun aber berichtete sie mit Tränen in den Augen, dass Ilse gestorben sei, eine Freundin ihrer Eltern in Deutschland, zu der sie, wie sich nun zeigte, ein ganz anderes Verhältnis hatte, als zu ihren Eltern. In weiteren Verlauf kam es zu einer heftigen Idealisierung von Ilse. Sie sprach ständig von ihr, lebte nur noch mit ihr und begann wieder mit Impulshandlungen. Die Patientin war weder mit Deutungen noch Einfühlung zu erreichen. Erst als Little in ihrer Verzweiflung sagte, dass sie nicht mehr weiter wüsste, dass es ihr leid tue, sie so zu sehen, begann sich grundlegend etwas zu ändern. Es war nun möglich, dass neben der Übertragung zur Mutter nun auch diejenige zu Ilse in der Analyse auftauchte.

Wie erklärt nun Lacan diesen Wandel? Er meint, dass diese Veränderung nicht primär etwas mit Symbolisierung zu tun habe und schon gar nicht etwa mit der Authentizität der Analytikerin, also damit, dass hier die realen Gefühle eingebracht werden, so nämlich interpretiert Little selbst diesen Prozess. Für Lacan hingegen geht es um etwas ganz anderes, nämlich das, was er die Funktion des Schnitts nennt. Er erklärt dies folgendermassen, dass es der Patientin möglich wird, eine Reaktion zu übertragen, eine Übertragung zu einer Person zu haben, zu machen, für die sie ein Mangel sein konnte, für die sie Bedeutung hat. Was die Analysandin in der Intervention der Analytikerin erkennen kann, dort wo diese an ihre Grenze kam, wo sie sagte, dass sie mit ihrem Latein am Ende sei, was sie dort erfahren konnte, ist die Angst und zugleich den Mangel der Analytikerin. Bislang konnte sich die Analysandin in keiner Beziehung als Objekt sehen: weder vom Vater noch von der Mutter war sie begehrt. Ganz in diesem Sinne interpretiert Lacan 
(1962/3: 146) dann die Kleptomanie aus der Sicht der Analysandin: «Ich zeige euch ein Objekt, das ich mit Gewalt und List geraubt habe und das bedeutet, dass es irgendwo ein anderes Objekt gibt, meines, das a, das, welches verdienen würde, dass man es in Betracht zieht, dass man es einen Augenblick lang sich isolieren lasse.» In dieser Isolierung, dieser Trennung, die sie, als Verlängerung der Mutter, nie haben konnte, nie kennen lernen konnte, liegt das Wesen der Funktion des Schnitts. In der Analytikerin macht sie die Erfahrung des Mangels, der Tatsache, dass diese von der Kastration gezeichnet ist, eine Grenze hat, begrenzt ist. Zudem macht sie die Erfahrung, für jemand Objekt der Angst, der Sorge zu sein, Erfahrungen, die bislang niemand für sie symbolisieren konnte, ausser vielleicht ansatzweise die erwähnte Ilse.

Ich habe diesen Fall immer beeindruckend gefunden und auch Lacans Interpretation; aber haben wir in dieser mehr als zehn Jahre dauernden Analyse/ Therapie nicht allem voran das, was man eine «corrective emotional experience» nennt, wenn Lacan davon spricht, dass die Analysandin sich aufgrund der Erfahrung des Mangels zum ersten Mal als Objekt der Angst, der Sorge erfahren konnte, ein erstes Mal abgetrennt. Also nochmal etwas anderes, nicht Analyse und auch nicht Suggestion? Lacan erklärt den Verlauf im Zusammenhang mit dem Objekt a, der Erfahrung des Mangels, Little hatte es mit ihrer Authentizität, ihren authentischen Reaktionen erklärt. Erklärungsmodelle mag es viele geben, und ihr Wert ergibt sich weitgehend aus der Pragmatik, wie sehr sie helfen, die Situation zu erfassen und Handlungsmöglichkeiten öffnen. Wesentlich an diesem Beispiel ist mir, dass wir hier, unabhängig von der dahinter liegenden Theorie, einen Prozess haben, in dem wir auf bewundernswerte Weise das Begehren, den Glauben einer Analytikerin am Werke sehen, der bewirkt, dass sowohl sie wie die Analysandin zur Sprache kommen; zugleich haben wir es mit einer Patientin zu tun, die dran bleibt, die nicht ablässt. Dabei beruht der Prozess wohl auf unterschiedlichsten Momenten, kann weder als rein therapeutisch, noch als rein analytisch verstanden werden. Vielmehr erfordert ein solcher Prozess, wie ihn Little mit dieser Patientin aufzeigt, die heute wohl als «Borderline» bezeichnet wird (vgl. Keul 2003), einiges; ich denke insbesondere an die Fähigkeit, sich in diesem Feld der Unschärfe, jenseits von Eindeutigkeiten und absolutem Wissen, somit zwischen Konstruktion und Dekonstruktion bewegen zu können. Damit müssen wir aber auch zur Kenntnis nehmen, dass eine solche Arbeit heute nicht selbstverständlich finanziert würde, sie könnte vielleicht auch sonst nicht durchgeführt werden, weil die Fähigkeit beider, die Ausbildung des Analytikers, allenfalls aber auch die Bereitschaft beider dazu nicht mehr da ist. Was demgegenüber Anerkennung finden würde, wären 
therapeutische Prozesse, die relativ nahe an konkreten, alltäglich Konflikten entlang führen würden.

Damit besteht das, was man heute als Pluralismus der Psychoanalyse bezeichnen muss, nicht allein in den verschiedenen Schulen, welche unterschiedliche Konzepte zum psychoanalytischen Geschehen entwickelt haben. Das nämlich, worin sich die Sichtweise auf den psychoanalytischen Prozess in Zukunft meines Erachtens vorab unterscheiden wird, bezieht sich auf andere Ebenen, und steht z. B. im Zusammenhang mit der Topik. Gemeint sind damit Prozesse, die einer «realeren», bewusstseinsnäheren oder einer unbewussteren Ebene angehören, damit auch mehr oder weniger beobacht-, mess- oder quantifizierbarer sind, vielleicht auch näher oder ferner sind von Prozessen, wie sie eine kognitive Verhaltentherapie ausmachen. Und viele dieser Prozesse werden wohl unter dem Label psychoanalytische Psychotherapie laufen und das zu einem schönen Teil wohl auch hier am PSZ.

Dies ist aber keineswegs die einzige Ebene, durch die sich die «unterschiedlichen Psychoanalysen» auszeichnen. In einer Art Nebensatz schreibt Kuster (in diesem Heft): «Wahrscheinlich muss man letztlich entscheiden, ob man sich lieber in der Erbfolge von Buchhaltern, Priestern oder Hypnotiseuren sieht.» Die Liste könnte, wenn auch nicht beliebig, so doch um einiges verlängert werden, ich denke z. B. an Pädagogen, an Juristen, Philosophen, vielleicht sogar an Heiler usw. Und jeder dieser Erben hat seine spezifische Form der geringeren oder grösseren Anlehnung, Tradierung, sein spezifisches Triebschicksal, und auch seine spezifische Ausrichtung, seinen Zugang, seine Leidenschaft in Bezug auf das psychoanalytische Geschehen. Buchhalter und Pfaffen wären dann zwei Typen, und diese passen unterschiedlich gut zu gewissen gesellschaftlichen Ansprüchen und leisten Anderes für die Psychoanalyse. Das, was ich zuvor als psychoanalytische Tradition darzustellen suchte, wäre wiederum eine anders geartete Ausrichtung, meine preferierte, eine, die sich mit etlichen zur Zeit geltenden gesellschaftlichen Spielregeln nicht gerade gut aussöhnen liesse, die aber als Forschung viel zum Verständnis dessen beitragen könnte, was hier und heute geschieht.

Und zum Schluss: eine weitere Differenz ergibt sich in einer Kultur des ständigen und flüchtigen Geniessens: die Tradition, unbezahlt die Arbeit zu erbringen, die es braucht, um an diesem Ort weiterhin Psychoanalyse zu vermittelt, ist Ausdruck einer Leidenschaft, die nicht ganz der heutigen Vermarktung entspricht. Solche Unterschiede gilt es in Bezug auf eine Zukunft der Psychoanalyse zu beachten, zu erhoffen wäre ein kreatives Spannungsfeld unterschiedlicher Zugänge und Traditionen, in dem sowohl Bindungen wie Brüche da sind und koexistieren. «Wir 
sind, wie früher so auch jetzt, immer bereit ... neues dazuzulernen», hat Freud (1918: 241)geschrieben. Ich hoffe, wir sind es auch.

\section{Literatur}

Boelderl, Arthur, R. (2002): «Bester in Religion». Über Jacques Lacans «Anders-alsGlauben». In: Riss 54/2.

Fischer, Anton (2008): Für die breiten Volksschichten können wir derzeit nichts tun oder der Psychoanalytiker zwischen potenziellem Bedarf und realer Nachfrage. Überlegungen zum ökonomischen Aspekt eines schwierigen Berufs in einem sich wandelnden politischen und ökonomischen Umfeld. In diesem Heft.

Freud, Sigmund (1915): Triebe und Triebschicksale. In: S. Freud: Studienausgabe Bd. III, Psychologie des Unbewussten, Fischer, Frankfurt.

Freud, Sigmund (1916): Die Übertragung. In: Freud, S.: Vorlesungen zur Einführung in die Psychoanalyse. Studienausgabe Bd. 1. Frankfurt/Main: Fischer, .

Freud, Sigmund (1916): Die analytische Therapie. In: Freud, S.: Vorlesungen zur Einführung in die Psychoanalyse. Studienausgabe. Frankfurt/Main: Fischer.

Freud, Sigmund (1919a]): Wege der psychoanalytischen Therapie. In: Freud, S.: Schriften zur Behandlungstechnik. Studienausgabe. Frankfurt/Main: Fischer.

Freud, Sigmund (1937): Die endliche und die unendliche Analyse. In: Behandlungstechnik, Ergänzungsband. Studienausgabe. Frankfurt/Main: Fischer.

Keul, Christoph (2003): Es geht um die Grenze, auf der sich der Platz des Mangels einrichtet. In: RISS 56: Themenheft Boderline, 39-68.

Kuster, Martin (2008): «Ich persönlich werde wohl die 〈klassischen〉 Analysen weitermachen ...» (Freud 1924). In: Journal für Psychoanalyse 49, 27-45.

Lacan, Jacques (1962/3): Seminar X: Die Angst. Zitiert nach einer Übersetzung von Gerhard Schmitz (1998).

Lacan, Jacques (1964): Seminar XI: Die vier Grundbegriffe der Psychoanalyse. WalterVerlag, Olten, 1978.

May, Ulrike (2007): Zur Dauer von Freuds Analysen. In: Psyche 6 und 7, 590-625.

Passett, Peter (2008): Ein Purist auf Abwegen. In: Journal für Psychoanalyse 49, 46-61.

Ricoeur, Paul (1969): Interpretation. Ein Versuch über Freud. 